\section{PTU-037 ENDOTHELIAL INJURY AND OXIDATIVE STRESS IN PATIENTS WITH SCHISTOSOMAL HEPATIC FIBROSIS: RELATION TO RENAL DYSFUNCTION AND HAEMODYNAMICS}

doi:10.1136/gutjnl-2012-302514c.37

${ }^{1} \mathrm{H}$ El Aggan, ${ }^{*}{ }^{2} \mathrm{M}$ Rashwan, ${ }^{3} \mathrm{~S}$ Abodeya, ${ }^{3} \mathrm{~S}$ Mahmoud. ${ }^{1}$ Department of Medicine, Faculty of Medicine, University of Alexandria, Alexandria, Egypt; ${ }^{2}$ Department of Cardiology, Faculty of Medicine, University of Alexandria, Alexandria, Egypt; ${ }^{3}$ Department of Medical Biochemistry, Faculty of Medicine, University of Alexandria, Alexandria, Egypt

Introduction Endothelial injury plays an important role in the pathogenesis of chronic renal diseases and may be related to oxidative stress. The present work was designed to study markers of endothelial injury and oxidative stress in patients with schistosomal hepatic fibrosis (SHF) in relation to renal dysfunction and haemodynamics.

Methods 45 patients with SHF and 15 healthy subjects were included in the study. The severity of liver disease was assessed using Child-Pugh scoring system. According to urinary albumin excretion rate, patients with SHF were classified into 15 patients with normoalbuminuria, 15 patients with microalbuminuria and 15 patients with persistent albuminuria. Endothelial injury was assessed by plasma von Willibrand factor (vWF) activity and serum ACE levels. Serum malondialdehyde (MDA) levels were also measured as a marker for oxidative stress. Tubular damage was determined by measuring 24-h urinary levels of leucine aminopeptidase (LAP). Renal haemodynamics were assessed using Duplex-doppler ultrasonography by calculating the diastolic/systolic renal flow velocity ratio $(\mathrm{d} / \mathrm{s})$, intrarenal renal resistive index $(\mathrm{RI})$ and hilar renal blood flow (RBF).

Results Compared to healthy subjects, patients with SHF showed significant increases in plasma vWF activity, serum levels of ACE and MDA and urinary LAP levels $(p<0.05)$. Serum MDA and urinary LAP levels were significantly higher in patients with microalbuminuria and persistent albuminuria than in patients with normoalbuminuria while serum ACE level was significantly higher in patients with persistent albuminuria than in those with normoalbuminuria or microalbuminuria $(p<0.05)$. Patients with SHF also showed a significant increase in RI and significant decreases in $d / s$ ratio and $R B F$ compared with healthy subjects regardless of the severity of glomerular injury $(p<0.05)$. No statistically significant correlations were found between the severity of liver disease on one hand and plasma vWF activity, serum ACE levels, urinary LAP levels and renal haemodynamics on the other hand in patients with SHF ( $p>0.05)$, while there was a significant positive correlation between serum MDA levels and Child-Pugh score in these patients $(\mathrm{p}<0.05)$.

Conclusion Endothelial injury, possibly due to oxidative stress, may play an important role in the pathogenesis of renal dysfunction and increased renovascular impedence in SHF and in the initiation of schistosomal nephropathy in this disease.

Competing interests None declared.

\section{PTU-038 LONG TERM FOLLOW-UP OF PATIENTS WITH NON-ALCOHOLIC STEATOHEPATITIS}

\section{doi:10.1136/gutjnl-2012-302514c.38}

${ }^{1} \mathrm{H}$ Lafferty, ${ }^{*} \mathrm{C} \mathrm{D}$ Evans, ${ }^{3} \mathrm{~K}$ A Oien, ${ }^{1} \mathrm{P}$ R Mills. ${ }^{1}$ Gastroenterology, Gartnavel General Hospital, Glasgow, UK; ${ }^{2}$ Gastroenterology, Borders General Hospital, Melrose, UK; ${ }^{3}$ Pathology, Glasgow Royal Infirmary, Glasgow, UK

Introduction Non-alcoholic steatohepatitis (NASH) is an increasingly common cause for liver disease. More evidence is needed to determine the natural history and prognosis of this condition. The aim of the study was to conduct a prospective follow-up of a group of patients with NASH confirmed on liver biopsy over a period of up to 26 years.

Methods A study of 62 patients who had NASH on liver biopsy performed during the period 1985 to 1994 was published in 2002. Biopsies were scored according to Brunt's system ${ }^{2}$ by a single pathologist. 59 of these patients were now traceable. Information on long-term outcome was obtained by review of case notes, electronic patient records and data from NHS Information Services Division and the Registrar General for Scotland. Follow-up duration was defined as the time between diagnosis by initial liver biopsy and last hospital attendance or death. 1 patient subsequently tested positive for hepatitis $\mathrm{C}$ and was excluded from further analysis.

Results 17 of the 58 patients were male, with an overall mean age at diagnosis of 44 (range 17-74 years). 56 were Caucasian and 2 Asian and mean follow-up in years was 15 (range 0-26). Mean body mass index at diagnosis was 27.6 (range 21.1-41.3). At diagnosis, 6 (10.3\%) had diabetes mellitus (DM), 14 (24.1\%) hypertension (HTN) and $4(6.9 \%)$ ischaemic heart disease (IHD). On follow-up a total of $21(36.2 \%)$ patients had a diagnosis of DM and $30(51.7 \%)$ HTN. On initial biopsy none of the patients had cirrhosis. During follow-up 15 (25.8\%) patients developed cirrhosis (3 biopsy proven and 12 clinical). 5 (8.6\%) patients developed hepatic decompensation with jaundice (2), ascites (2), encephalopathy (1), variceal haemorrhage (1) and hepatoma (1). 28 (48.3\%) of the patients have died at a mean age of 70.4 years (range 33-97). Time to death from diagnosis was 0.5 to 24 years (mean 14). Two patients died of liver failure (7.1\%), 4 from non-hepatic malignancy (14.3\%), 6 from IHD (21.4\%), 3 of infectious causes $(10.7 \%), 3$ of cerebrovascular disease $(10.7 \%)$ and 10 of "other causes" (2 chronic obstructive pulmonary disease, 1 DM, 1 alcohol, 1 pancreatitis, 5 unknown).

Conclusion This observational study shows that the outcome of $\mathrm{NASH}$ is not benign with a significant mortality over a mean of 15 years, largely due to vascular disease and malignancy as expected. Progression to cirrhosis occurred in $26 \%$ of patients causing considerable clinical morbidity, but death from liver disease over this time period was uncommon.

Competing interests None declared.

\section{REFERENCES}

1. J Clin Path 2002;55:689-692.

2. Am J Gastroenterol 1999;94:2467-74

\section{PTU-039 MORTALITY AND UTILITY OF PROGNOSTIC SCORING MODELS IN CIRRHOTIC PATIENTS ADMITTED TO A TERTIARY NON-TRANSPLANT INTENSIVE CARE UNIT (ICU) IN THE UK}

doi:10.1136/gutjnl-2012-302514c.39

${ }^{1} \mathrm{H}$ Lewis, ${ }^{2} \mathrm{~T}$ Reynolds, ${ }^{3} \mathrm{~A}$ Lillis, ${ }^{3} \mathrm{~K}$ Maitland, ${ }^{1} \mathrm{G}$ R Foster, ${ }^{3} \mathrm{~J}$ Hadley. ${ }^{1}$ The Liver Unit, Queen Mary University of London, London, UK; ${ }^{2}$ Anaesthetics, Newham University Hospital, London, UK; ${ }^{3}$ Intensive Care, Barts and the London NHS Trust, London, UK

Introduction Patients with liver cirrhosis admitted to ICU have a poor prognosis with reported ICU, in-hospital and 1-year mortality rates of $37 \%>65 \%, 49-74 \%$ and $69-81 \%$ respectively. Prognostic models may enable accurate discrimination at admission of those who will benefit from ICU admission. Organ failure scoring models have been shown to be good predictors of ICU mortality, but there are few reports of their utility in non-transplant centres in Europe. We aimed to describe mortality and the prognostic value of commonly used predictors in patients with cirrhosis admitted to the ICU of a tertiary non-transplant London teaching hospital ICU. 